\title{
Determination of Pesticide Residues by GC-MS Using Analyte Protectants to Counteract the Matrix Effect
}

\author{
Consuelo Sánchez-Brunete, Beatriz Albero, Germán Martín, and José L. TAdeO \\ Departamento de Medio Ambiente, Instituto Nacional de Investigación y Tecnología Agraria y \\ Alimentaria (INIA), Carretera de la Coruña Km 7, 28040 Madrid, Spain
}

\begin{abstract}
An analytical method was developed to determine pesticides of various chemical classes in soil, juice and honey using analyte protectants to counteract the enhancement of the chromatographic response produced by the presence of matrix components (matrix effect). This effect was more pronounced for soil and honey samples than for juice samples; regarding the pesticide chemical class, organochlorine pesticides were less affected by the presence of matrix components than triazines and organophosphorus pesticides. Several analyte protectants (2,3-butanediol, L-gulonic acid $\gamma$-lactone, corn oil and olive oil) were tested for counteracting the observed matrix effect. L-Gulonic acid $\gamma$-lactone was an effective protecting agent for most of the pesticides studied in soil and honey samples, whereas olive oil was very effective for juice samples. The combination of these two protectants was found to be an effective analyte protectant for all compounds in soil and honey samples.
\end{abstract}

(Received April 4, 2005; Accepted June 6, 2005)

\section{Introduction}

Pesticide residues have generally been analyzed by gas liquid chromatography (GC) with different detectors, such as nitrogenphosphorus (NPD), electron-capture (ECD), and mass spectrometry (MS). ${ }^{1,2}$ In GC analysis, some analytes can give peaks showing a low response or poor peak shapes that are difficult to integrate. These problems can be originated by partial degradation of the analyte at the high temperature of the injection port, or by a low transference to the chromatographic column due to its retention in the active sites of the injector. Nevertheless, the sample components may compete for the active sites in the glass liner, decreasing the interaction between the active sites and the analyte, and thus allowing a larger amount of analyte to be transferred to the chromatographic column. This fact, known as the matrix-induced chromatographic response effect (matrix effect), has been studied in relation to diverse parameters, such as sample cleanup $^{3}$ or injection techniques.,5 Moreover, the matrix effect is also described as one of the main sources of errors in multiresidue analytical methods. ${ }^{6,7}$ Additional sample clean-up can compensate for the chromatographic response enhancement due to removal of the matrix components that cause this effect; but this is not always possible, and this approach increases the time and labor in sample preparation, and may produce analyte losses, leading to a higher uncertainty in the results. In general, this problem has been overcome by the method of standard addition in blanks, ${ }^{8-13}$ although this solution has the drawback of needing blank sample extracts for calibration standards. Furthermore, some official organizations, such as the U.S. federal agencies, do not permit the use of blank fortified standards in analyses. Another way to solve the matrix effect

$\dagger$ To whom correspondence should be addressed.

E-mail: tadeo@inia.es problem is the use of analyte protectants. These compounds, added to the standards, may protect analytes from degradative interactions, which can take place in the injection port. Several authors have demonstrated that this kind of additive was capable of counteracting, in some extension, the matrix effect. ${ }^{11,14,15}$

The aim of this work was to develop an analytical method for the determination of pesticide residues in environmental (soil) and food (juice and honey) samples using analyte protectants to counteract the matrix effect. Four analyte protectants (2,3butanediol, L-gulonic acid $\gamma$-lactone, corn oil and olive oil) were evaluated for pesticide determination in the studied samples.

\section{Experimental}

\section{Chemicals and reagents}

Pesticide standards were obtained from Reidel-de Haën (Seelze, Germany), and all compounds were of 99\% purity. Ethyl acetate, hexane, methanol, acetonitrile and acetone, residue analysis grade, were purchased from Scharlab (Barcelona, Spain). A Milli-Q water purification system from Millipore (Bedford, MA) was used to provide ultrapure water. Silica Bondesil- $\mathrm{C}_{18}$, particle diameter of $40 \mu \mathrm{m}$, was acquired from Scharlab (Barcelona, Spain). The compounds evaluated as protecting agents were L-gulonic acid $\gamma$-lactone from Fluka (Buchs, Germany) and 2,3-butanediol from Aldrich (Milwaukee, WI). Corn and olive oils were purchased in a local supermarket.

Stock solutions $(500 \mu \mathrm{g} / \mathrm{ml})$ of each pesticide standard were prepared in ethyl acetate and stored at $4^{\circ} \mathrm{C}$. A standard stock solution $(5 \mu \mathrm{g} / \mathrm{ml})$ containing all of the pesticides was prepared in ethyl acetate. Working solutions were prepared by appropriate dilution of the stock solution. The internal standard was prepared by dissolving hexachlorobenzene (HCB) in ethyl acetate to make a $500 \mu \mathrm{g} / \mathrm{ml}$ solution. A stock solution of L- 
Table 1 Operational conditions of GC-MS

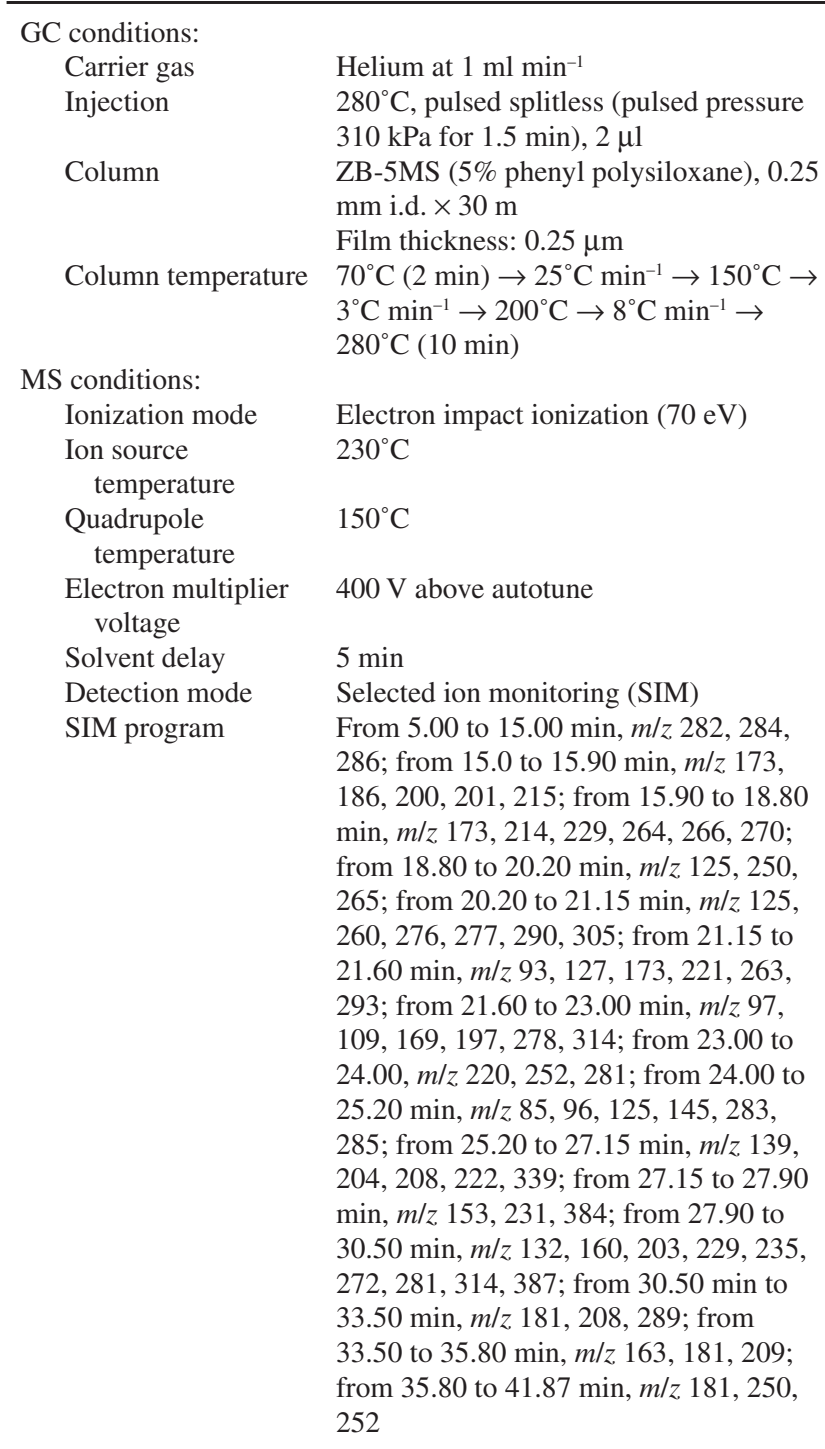

gulonic acid $\gamma$-lactone $(10 \mathrm{mg} / \mathrm{ml})$ was prepared in acetone-water $(8: 2, \mathrm{v} / \mathrm{v})$ using an ultrasonic bath. Stock solutions $(1 \%, \mathrm{v} / \mathrm{v})$ of the other three analyte protectants, 2,3butanediol, corn oil and olive oil, were prepared in ethyl acetate.

\section{Samples}

Food samples, orange honey and apple juice, were purchased from supermarkets in Madrid. The samples were stored at $4{ }^{\circ} \mathrm{C}$ until analysis. Soil used in the study was collected from the plough layer $(0-10 \mathrm{~cm})$ of an experimental plot located in the region of Madrid (Spain). Soil was sieved ( $2 \mathrm{~mm}$ ) and stored at room temperature. The characteristics of the selected soil was: $\mathrm{pH}, 7.69$; organic matter content, $0.97 \%$; sand, $44.34 \%$; silt, $37.44 \%$; and clay, $18.22 \%$. These food and soil samples were analyzed, and no pesticide residues were found.

\section{Instrumentation}

An Agilent gas chromatograph (Model 6890) equipped with an automatic split-splitless injector (Model HP 7683) and a mass spectrometric detector (MSD; Model HP 5973) were used in the chromatographic analysis. The operating conditions are summarized in Table 1.
Table 2 Retention time $\left(t_{\mathrm{R}}, \min \right)$, molecular weight $\left(M_{\mathrm{W}}\right)$, target ion $(\mathrm{T})$, qualifier ions $\left(\mathrm{Q}_{1}, \mathrm{Q}_{2}\right)$, and qualifier to target abundance ratios $\left(\mathrm{Q}_{1} / \mathrm{T}, \mathrm{Q}_{2} / \mathrm{T}\right)^{\mathrm{a}}$ of the studied pesticides

\begin{tabular}{lccccccc}
\hline \multicolumn{1}{c}{ Pesticide } & $t_{\mathrm{R}}$ & $M_{\mathrm{W}}$ & $\mathrm{T}$ & $\mathrm{Q}_{1}$ & $\mathrm{Q}_{2}$ & $\mathrm{Q}_{1} / \mathrm{T}, \%$ & $\mathrm{Q}_{2} / \mathrm{T}, \%$ \\
\hline HCB (IS) & 14.71 & 284.8 & 284 & 286 & 282 & 100.0 & 100.0 \\
Simazine & 15.27 & 201.7 & 201 & 186 & 173 & 66.8 & 60.0 \\
Atrazine & 15.51 & 215.7 & 200 & 215 & 173 & 64.2 & 22.2 \\
Terbuthylazine & 16.20 & 229.7 & 214 & 229 & 173 & 31.4 & 40.1 \\
Chlorothalonil & 17.41 & 265.9 & 266 & 264 & 270 & 100.0 & 100.0 \\
Tolclofos-methyl & 19.45 & 301.1 & 265 & 125 & 250 & 14.8 & 10.1 \\
Fenitrothion & 20.76 & 277.2 & 277 & 125 & 260 & 93.5 & 37.8 \\
Pirimiphos-methyl & 20.92 & 333.4 & 290 & 276 & 305 & 76.1 & 62.4 \\
Aldrin & 21.31 & 364.9 & 263 & 293 & 221 & 36.8 & 20.7 \\
Malathion & 21.43 & 330.4 & 173 & 127 & 93 & 79.3 & 51.1 \\
Fenthion & 21.82 & 278.3 & 278 & 169 & 109 & 23.7 & 20.4 \\
Chlorpyrifos & 21.93 & 350.6 & 197 & 314 & 97 & 74.9 & 48.4 \\
Pendimethalin & 23.53 & 281.3 & 252 & 281 & 220 & 11.6 & 9.10 \\
Procymidone & 24.31 & 284.1 & 283 & 96 & 285 & 89.3 & 100.0 \\
Methidathion & 24.61 & 302.3 & 145 & 85 & 125 & 52.4 & 20.3 \\
Profenophos & 25.84 & 373.6 & 208 & 339 & 139 & 67.9 & 86.4 \\
Cyproconazole & 26.72 & 291.8 & 222 & 139 & 204 & 48.8 & 35.9 \\
Ethion & 27.56 & 384.5 & 231 & 153 & 384 & 43.0 & 13.1 \\
Ofurace & 28.12 & 281.7 & 132 & 160 & 281 & 80.8 & 54.1 \\
Endosulfan sulfate & 28.36 & 423.0 & 272 & 229 & 387 & 72.9 & 48.5 \\
Nuarimol & 28.91 & 314.7 & 235 & 203 & 314 & 62.6 & 52.0 \\
Acrinathrin & 31.71 & 541.4 & 181 & 208 & 289 & 128.0 & 54.7 \\
$\alpha$-Cypermethrin & 34.22 & 416.3 & 181 & 163 & 209 & 106.4 & 175.7 \\
Fluvalinate tau- I & 36.24 & 502.9 & 250 & 181 & 252 & 32.0 & 100.0 \\
Fluvalinate tau-II & 36.38 & 502.9 & 250 & 181 & 252 & 40.1 & 100.0 \\
\hline
\end{tabular}

a. Q/T $(\%)$ are the results of abundance values of the qualifier ion $(\mathrm{Q} 1, \mathrm{Q} 2)$ divided by the abundance of the target ion $(\mathrm{T}) \times 100$.

b. IS: Internal standard.

\section{Extraction procedure}

The extraction procedures used for pesticide determination in soil and food samples were based on previously published methods. ${ }^{8-10}$

Soil: A polypropylene column $(20 \mathrm{ml})$, with two Whatman No. 1 filter paper circles of $2 \mathrm{~cm}$ of diameter at the bottom end, was filled with anhydrous sodium sulfate $(2 \mathrm{~g})$ and sieved soil $(10 \mathrm{~g})$. Samples were extracted twice with $5 \mathrm{ml}$ of ethyl acetate for $15 \mathrm{~min}$ in an ultrasonic water bath (Raypa, Spain) at room temperature. After each extraction, the columns were placed on a 12-port vacuum manifold (Supelco Visiprep, Madrid, Spain), where the solvent was filtered and collected in graduated tubes.

Honey: A $10 \mathrm{~g}$ amount of honey was dissolved in $10 \mathrm{ml}$ of water-methanol (70:30) in a Sovirell tube. A polypropylene column $(5 \mathrm{ml})$, with a polyethylene frit of $1 \mathrm{~cm}$ diameter and 20 $\mu \mathrm{m}$ pore size (Varian, USA), was filled with $1 \mathrm{~g}$ of $\mathrm{C}_{18}$ and placed on a 12-port vacuum manifold. The column was preconditioned with $3 \mathrm{ml}$ of acetonitrile and $5 \mathrm{ml}$ of water. The honey solutions were transferred to the column and the Sovirell tube was washed with $5 \mathrm{ml}$ of water-methanol (70:30), which were also transferred to the column. The column was then eluted twice with $5 \mathrm{ml}$ of hexane-ethyl acetate $(1: 1, \mathrm{v} / \mathrm{v})$, and the extracts were collected in $10 \mathrm{ml}$ graduated tubes.

Juice: A $10 \mathrm{ml}$ volume of juice was placed in a Sovirell tube and $3 \mathrm{ml}$ of methanol was added. The extraction columns (5 $\mathrm{ml}$ ), filled with $1 \mathrm{~g}$ of $\mathrm{C}_{18}$, were preconditioned with $3 \mathrm{ml}$ of acetonitrile and $5 \mathrm{ml}$ of water using a 12-port vacuum manifold to filter the solvents. The juice solution was transferred to the column and the Sovirell tube was washed with $5 \mathrm{ml}$ of water, which was also transferred to the column. The elution was carried out twice with $5 \mathrm{ml}$ of hexane-ethyl acetate $(1: 1, \mathrm{v} / \mathrm{v})$, 
and the eluates were collected in $10 \mathrm{ml}$ graduated tubes. A $0.5 \mathrm{ml}$ volume of a $1 \mu \mathrm{g} / \mathrm{ml}$ solution of the internal standard (HCB) was added before GC-MS analysis.

\section{Determination of pesticides by $G C-M S$}

Analyses were performed with selected ion monitoring (SIM) using one target and two qualifier ions. The target and qualifier abundances were determined by the injection of individual pesticide standards under the same chromatographic conditions using a full scan with the mass/charge ratio ranging from 60 to $500 \mathrm{~m} / \mathrm{z}$. Quantitation was based on the ratio of the peak area of the target ion divided by the peak area of the internal standard in samples versus that found in the calibration standard. Table 1 shows the SIM program used to determine and confirm pesticides, and Table 2 lists the pesticides along with their retention times, the target and qualifier ions and their qualifier to target abundance ratios. Pesticides were confirmed by their retention times, the identification of target and qualifier ions and the determination of qualifier to target ratios. The retention times had to be within $\pm 0.2 \mathrm{~min}$ of the expected time and the qualifier-to-target ratios had to be within a $20 \%$ range for positive confirmation. Quantitation was performed and compared by using calibrations standards involving standards in a neat solvent, standards added to blank extracts, and standards in solutions containing analyte protectants. The blank extracts were fortified with $0.5 \mathrm{ml}$ of the pesticide standard solution and $0.5 \mathrm{ml}$ of the internal standard solution.

\section{Results and Discussion}

\section{Matrix effect}

In previous work, an enhancement of the chromatographic response was observed when blank soil or a food sample was spiked with known amounts of pesticides. ${ }^{8-11}$ Compounds with hydroxy $(-\mathrm{OH})$ or amino groups (R-NH-), azoles $(-\mathrm{N}=)$, and several organophosphorus pesticides (OP) have been found to be more susceptible to the matrix effect, which is also influenced by the actual chromatographic conditions, particularly those of the injection port and the head of the column. Figure 1A shows chromatograms obtained for a fortified blank standard and a matrix-free standard. In general, a response increase of around two-fold was observed, although simazine and ofurace showed a higher effect, and three compounds were not detected in the matrix free standard. Organochlorine pesticides (OC) were compounds that presented the lowest matrix effect. These compounds (OC) are less polar, and hence are less susceptible to adsorption by the active sites in the injection liner. The chromatographic response enhancement observed in our study has been reported by other authors in the determination of pesticides in food commodities. ${ }^{3,4,14}$ Figure 1A shows that some organophosphorus $(\mathrm{P}=\mathrm{O})$ pesticides (fenitrothion, fenthion and sometimes methidathion) were not detected when injected in neat solvent, although for some organophosphorus containing $\mathrm{P}=\mathrm{S}$ groups, ethion and malathion, the incidence of matrix effect was less pronounced. Other compounds, such as simazine and ofurace containing a $\mathrm{NH}$ group, gave high values due to the low response obtained in neat solvent standards. In the case of synthetic pyrethroids, compounds with high molecular masses (over $400 \mathrm{~g} / \mathrm{mol}$ ), they generally gave in neat solvent standards poor peak shapes that were more difficult to integrate and originate a response increase around two-fold in matrix fortified standards.
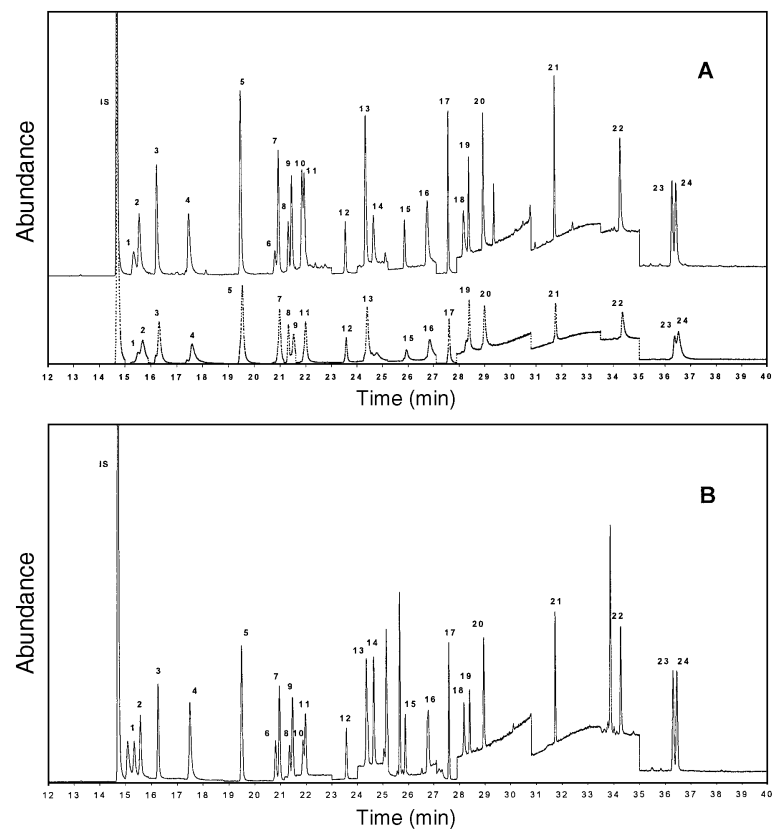

Fig. 1 GC-MS-SIM chromatograms: (A) blank juice extract fortified at $50 \mu \mathrm{g} / \mathrm{l}$ (unbroken line) and a pesticide standard in ethyl acetate at $50 \mu \mathrm{g} / \mathrm{l}$ (dotted line); (B) pesticide standard at $50 \mu \mathrm{g} / \mathrm{l}$ prepared in ethyl acetate with $0.2 \%$ of olive oil and $0.2 \mathrm{mg} / \mathrm{ml}$ of Lgulonic acid $\gamma$-lactone. Peak identification: IS, internal standard; 1 , simazine; 2 , atrazine; 3 , terbuthylazine; 4 , chlorothalonil; 5 , tolclofosmethyl; 6, fenitrothion; 7, pirimiphos-methyl; 8 , aldrin; 9, malathion; 10 , fenthion; 11, chlorpyrifos; 12 , pendimethalin; 13, procymidone; 14, methidathion; 15 , profenofos; 16 , cyproconazole; 17 , ethion; 18 , ofurace; 19 , endosulfan sulfate; 20, nuarimol; 21 , acrinathrin; $22, \alpha$ cypermethrin; 23, fluvalinate tau-I; 24, fluvalinate tau-II.

\section{Analyte protectants}

The addition of analyte protectants in order to mask active sites in the injection port and protect pesticides from degradative interactions has been previously assayed. ${ }^{11}$ Ideally, the analyte protectant should provide the same degree of protection (same peak area) as that of the solution containing the matrix components. Several factors have to be considered in selecting the analyte protectants, such as their volatility and hydrogen-bonding ability. Based on previous studies, 2,3butanediol, L-gulonic acid $\gamma$-lactone ${ }^{11}$ and corn oil ${ }^{16}$ were selected as protectant agents. Olive oil was also included in the study. These compounds were added individually or in combination to a mixture of pesticides in ethyl acetate. A compound with several hydroxy groups, such as 2,3-butanediol, could be an effective analyte protectant, since these groups could interact with the silanol groups of the glass liner and the column head, masking the active sites, and thus increasing the analyte transfer from the injection port to the column. L-Gulonic acid $\gamma$-lactone, a sugar derivative, was shown to be very effective in a previous study. ${ }^{11}$ This compound together with its degradation products cover a wide range of volatility, and therefore protects pesticides with different retention times without interfering with the chromatographic analysis. Corn and olive oils were selected as analyte protectants due to the presence of long-chain fatty acids that may reduce the degradation of base-sensitive pesticides.

The concentration of the analyte protecting agent in the standard solution is an important factor in the response enhancement effect. To determine the effect of the protectant 
Table 3 Pesticide quantification in fortified blank samples using standards prepared in ethyl acetate with (A) $0.2 \mathrm{mg} / \mathrm{ml}$ of L-gulonic acid $\gamma$-lactone and (B) $0.2 \%$ of olive oil. The results are expressed in $\%$ of the added amount (50 ppb) \pm relative standard deviation (RSD)

\begin{tabular}{|c|c|c|c|c|c|c|}
\hline & \multicolumn{2}{|c|}{ Soil } & \multicolumn{2}{|c|}{ Juice } & \multicolumn{2}{|c|}{ Honey } \\
\hline & A & $\mathrm{B}$ & A & $\mathrm{B}$ & $\mathrm{A}$ & $\mathrm{B}$ \\
\hline Simazine & $71.0 \pm 2.7$ & $156.3 \pm 3.2$ & $65.9 \pm 2.0$ & $109.5 \pm 10.2$ & $115.0 \pm 5.5$ & $236.8 \pm 8.4$ \\
\hline Atrazine & $81.7 \pm 1.0$ & $109.1 \pm 4.5$ & $81.7 \pm 1.6$ & $102.1 \pm 5.5$ & $99.6 \pm 4.1$ & $126.9 \pm 7.0$ \\
\hline Terbuthylazine & $82.7 \pm 2.1$ & $104.0 \pm 2.4$ & $86.7 \pm 1.2$ & $102.6 \pm 3.3$ & $94.7 \pm 1.4$ & $113.8 \pm 1.7$ \\
\hline Chlorothalonil & $77.5 \pm 1.3$ & $129.1 \pm 5.3$ & $72.4 \pm 3.2$ & $108.5 \pm 6.5$ & $99.6 \pm 1.5$ & $149.4 \pm 5.5$ \\
\hline Tolclofos-methyl & $88.4 \pm 0.4$ & $104.9 \pm 2.6$ & $90.1 \pm 1.3$ & $103.3 \pm 3.5$ & $99.5 \pm 2.3$ & $112.9 \pm 3.0$ \\
\hline Fenitrothion & $71.1 \pm 2.6$ & $185.3 \pm 3.0$ & $52.4 \pm 6.7$ & $94.8 \pm 10.2$ & $115.4 \pm 2.6$ & $264.4 \pm 4.0$ \\
\hline Pirimiphos-methyl & $88.9 \pm 0.5$ & $104.5 \pm 3.0$ & $88.8 \pm 3.1$ & $102.4 \pm 6.5$ & $104.6 \pm 4.4$ & $111.1 \pm 4.3$ \\
\hline Aldrin & $94.8 \pm 1.5$ & $100.1 \pm 2.0$ & $95.5 \pm 1.3$ & $102.8 \pm 2.4$ & $99.5 \pm 2.3$ & $105.1 \pm 1.8$ \\
\hline Malathion & $88.9 \pm 1.7$ & $110.5 \pm 10.3$ & $83.2 \pm 3.1$ & $104.5 \pm 7.7$ & $105.4 \pm 6.5$ & $126.5 \pm 7.2$ \\
\hline Fenthion & $91.1 \pm 5.0$ & $114.8 \pm 5.4$ & $89.5 \pm 0.4$ & $110.2 \pm 8.1$ & $101.5 \pm 10.3$ & $120.4 \pm 6.6$ \\
\hline Chlorpyrifos & $96.9 \pm 2.1$ & $110.1 \pm 3.7$ & $93.1 \pm 2.7$ & $101.8 \pm 5.8$ & $97.0 \pm 3.4$ & $104.6 \pm 4.2$ \\
\hline Pendimethalin & $96.9 \pm 1.0$ & $117.5 \pm 7.2$ & $84.7 \pm 3.6$ & $104.1 \pm 8.1$ & $103.2 \pm 3.3$ & $113.5 \pm 4.7$ \\
\hline Procymidone & $92.1 \pm 1.1$ & $106.9 \pm 4.3$ & $91.3 \pm 3.6$ & $98.3 \pm 7.2$ & $105.9 \pm 3.2$ & $114.4 \pm 3.0$ \\
\hline Methidathion & $83.1 \pm 2.3$ & $144.1 \pm 13.5$ & $70.9 \pm 2.5$ & $99.4 \pm 5.8$ & $106.3 \pm 7.9$ & $152.5 \pm 7.4$ \\
\hline Profenofos & $84.6 \pm 11.3$ & $108.7 \pm 8.2$ & $89.9 \pm 9.5$ & $99.0 \pm 10.8$ & $101.4 \pm 10.6$ & $118.1 \pm 5.1$ \\
\hline Cyproconazole & $82.8 \pm 3.0$ & $106.8 \pm 6.2$ & $78.5 \pm 6.4$ & $100.9 \pm 10.8$ & $100.8 \pm 8.9$ & $114.8 \pm 2.2$ \\
\hline Ethion & $89.0 \pm 7.0$ & $109.9 \pm 8.7$ & $87.7 \pm 10.1$ & $105.5 \pm 11.7$ & $96.7 \pm 3.1$ & $113.3 \pm 7.8$ \\
\hline Ofurace & $91.9 \pm 4.5$ & $133.8 \pm 3.5$ & $67.1 \pm 5.7$ & $100.4 \pm 1.9$ & $96.9 \pm 7.6$ & $137.4 \pm 12.0$ \\
\hline Endosulfan sulfate & $97.7 \pm 0.7$ & $111.9 \pm 4.3$ & $95.3 \pm 3.6$ & $106.2 \pm 9.6$ & $102.2 \pm 3.2$ & $107.5 \pm 3.4$ \\
\hline Nuarimol & $94.8 \pm 1.8$ & $107.9 \pm 5.5$ & $92.7 \pm 1.9$ & $100.9 \pm 10.2$ & $101.5 \pm 5.0$ & $109.0 \pm 5.9$ \\
\hline Acrinathrin & $96.4 \pm 3.5$ & $103.4 \pm 5.8$ & $101.9 \pm 7.4$ & $99.8 \pm 10.7$ & $104.4 \pm 3.2$ & $107.5 \pm 7.4$ \\
\hline$\alpha$-Cypermethrin & $103.6 \pm 7.1$ & $109.4 \pm 7.2$ & $89.3 \pm 2.9$ & $87.8 \pm 4.8$ & $103.6 \pm 1.7$ & $102.3 \pm 5.9$ \\
\hline Fluvalinate tau-I & $526.6 \pm 5.9$ & $99.2 \pm 3.9$ & $403.2 \pm 2.3$ & $76.0 \pm 4.7$ & $521.1 \pm 4.4$ & $100.3 \pm 7.0$ \\
\hline Fluvalinate tau-II & $210.6 \pm 9.8$ & $93.0 \pm 2.2$ & $222.0 \pm 8.8$ & $81.7 \pm 3.4$ & $216.4 \pm 10.6$ & $96.4 \pm 7.3$ \\
\hline
\end{tabular}

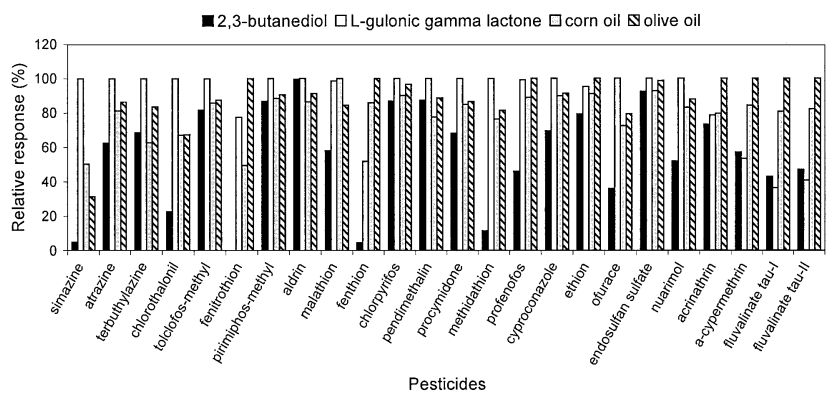

Fig. 2 Comparison of the relative responses of the selected pesticides at $50 \mathrm{ppb}$, in ethyl acetate, obtained with each analyte protectant. The results are expressed as percentage of the maximum relative response to the internal standard obtained for each pesticide with the different analyte protectants at a concentration of $0.2 \mathrm{mg} / \mathrm{ml}$ for L-gulonic acid $\gamma$-lactone and $0.2 \%$ for the other analyte protectants.

concentration, different standard solutions containing $0.1,0.2$, and $0.3 \%$ of 2,3-butanediol, corn oil, or olive oil and standard solutions containing $0.1,0.2$, and $0.3 \mathrm{mg} / \mathrm{ml}$ of L-gulonic acid $\gamma$ lactone were assayed. For the lowest concentration of analyte protectant added, no effect was observed for some of the pesticides, such as fenitrothion and methidathion. For the other two concentrations of analyte protectant assayed, a high and similar increase in the response was obtained. When a higher concentration of protectant $(0.5 \%$ or $0.5 \mathrm{mg} / \mathrm{ml})$ was studied, the increase in the response was not improved and, therefore, the intermediate concentration level $(0.2 \%$ or $0.2 \mathrm{mg} / \mathrm{ml})$ of the analyte protectant was selected.

Figure 2 shows a comparison of the relative responses to the internal standard of selected pesticides at $50 \mathrm{ppb}$ obtained with each analyte protectant expressed as the percentage of the maximum relative response achieved with any of the protectants. Therefore, $100 \%$ represents the protectant that gave the highest relative response to the internal standard for each pesticide. The analyte protectant that showed the lowest chromatographic response enhancement effect in most of the pesticides studied was 2,3-butanediol, which is in agreement with previous findings. ${ }^{9}$ This analyte protectant is the most volatile agent assayed, and its protection for the late-eluting peaks was, in general, lower than for those with shorter retention times. L-gulonic acid $\gamma$-lactone provided good protection for almost all of the pesticides analyzed, due to the wide range of volatility covered by its degradation products. Nevertheless, the protection provided by this agent to the lateeluting pyrethroids was inadequate, whereas olive and corn oils gave good protection to those compounds. This may be explained by the presence of long-chain fatty acids that cover a volatility range similar to that of the analytes and minimize, at the same time, the degradation caused by active sites to the pyrethroids, which are base-sensitive compounds. Other authors ${ }^{16}$ have reported good recovery results in wine samples when calibration standards were prepared with corn oil as an analyte protectant. These facts suggest that the volatility range covered by the mixture of fatty acids gives good analyte protection despite differences in the composition of corn and olive oils. Although in our assay the behavior of both oils was similar, better results were obtained, in general, with olive oil, and therefore this oil was selected for further studies.

\section{Determination of pesticide residues with analyte protectants}

Three types of matrices (soil, fruit juice and honey), which have different contents of moisture and organic components, were employed for the determination of pesticide residues. The pesticides chosen covered a wide range of polarity, volatility and other physicochemical properties. Several pesticides, such as organochlorine pesticides, were slightly affected, whereas 
Table 4 Determination of pesticide residues with analyte protectants $^{\mathrm{a}}$

\begin{tabular}{lrr}
\hline \multirow{2}{*}{ Pesticide } & \multicolumn{2}{c}{ Recovery $(\%) \pm$ RSD $(\%)$} \\
\cline { 2 - 3 } & \multicolumn{1}{c}{ Soil } & \multicolumn{1}{c}{ Honey } \\
\hline Simazine & $74.0 \pm 2.7$ & $106.3 \pm 2.7$ \\
Atrazine & $82.9 \pm 2.2$ & $96.4 \pm 2.1$ \\
Terbuthylazine & $84.3 \pm 1.6$ & $95.1 \pm 1.2$ \\
Chlorothalonil & $78.6 \pm 1.4$ & $93.6 \pm 1.4$ \\
Tolclofos-methyl & $91.4 \pm 2.4$ & $99.2 \pm 2.3$ \\
Fenitrothion & $71.7 \pm 1.6$ & $98.0 \pm 2.6$ \\
Pirimiphos-methyl & $92.6 \pm 4.2$ & $100.4 \pm 3.1$ \\
Aldrin & $94.0 \pm 1.5$ & $99.7 \pm 2.0$ \\
Malathion & $92.6 \pm 2.8$ & $100.6 \pm 4.2$ \\
Fenthion & $89.5 \pm 2.4$ & $86.2 \pm 0.8$ \\
Chlorpyrifos & $98.6 \pm 2.2$ & $91.3 \pm 3.3$ \\
Pendimethalin & $98.6 \pm 2.9$ & $99.8 \pm 2.1$ \\
Procymidone & $95.9 \pm 3.3$ & $107.4 \pm 1.8$ \\
Methidathion & $85.7 \pm 2.9$ & $100.4 \pm 2.3$ \\
Profenofos & $92.7 \pm 4.9$ & $100.9 \pm 2.6$ \\
Cyproconazole & $89.7 \pm 2.9$ & $97.1 \pm 2.1$ \\
Ethion & $95.0 \pm 6.6$ & $101.3 \pm 3.8$ \\
Ofurace & $93.1 \pm 8.2$ & $95.6 \pm 6.5$ \\
Endosulfan sulfate & $100.8 \pm 6.1$ & $101.6 \pm 2.8$ \\
Nuarimol & $94.6 \pm 6.2$ & $95.9 \pm 1.5$ \\
Acrinathrin & $86.8 \pm 4.2$ & $98.7 \pm 7.1$ \\
$\alpha$-Cypermethrin & $82.3 \pm 5.5$ & $84.1 \pm 5.0$ \\
Fluvalinate tau-I & $80.6 \pm 3.0$ & $76.7 \pm 4.5$ \\
Fluvalinate tau-II & $80.0 \pm 2.1$ & $75.2 \pm 4.7$ \\
\hline
\end{tabular}

a. Calibration standard $(50 \mathrm{ppb})$ prepared in ethyl acetate with 0.2 $\mathrm{mg} / \mathrm{ml}$ of L-gulonic acid $\gamma$-lactone and $0.2 \%$ of olive oil. Samples were fortified at $50 \mathrm{ppb}$.

some pesticides, such as synthetic pyrethroids, gave narrow and tall peaks, and other pesticides, such as some organophosphorus, gave peaks four times higher when injected with analyte protectants. To study the determination of pesticide residues in the three different matrices chosen, pesticide-free sample extracts were fortified with a mixture of pesticides. Pesticide residues in the studied matrices, extracted following the procedures described above, were quantified using calibration standards containing the analyte protectants at $0.2 \%$ or $0.2 \mathrm{mg} / \mathrm{ml}$, individually or in combination.

Table 3 gives the results for the quantification of pesticides in fortified soil, juice and honey samples using calibration standards prepared in ethyl acetate with L-gulonic acid $\gamma$-lactone or olive oil. L-Gulonic acid $\gamma$-lactone gave, in general, good quantification results for all pesticides studied, except for fluvalinate in soil, juice and honey, where the matrix effect was not counteracted. Olive oil gave good results for all pesticides in juice samples, whereas in soil and honey samples the quantification results were good for all pesticides, except for some OP, such as fenitrothion and methidathion, and other compounds, such as simazine and ofurace, where the response enhancement was not completely counteracted by the protectant.

Therefore, due to the complementary effect obtained with both analyte protectants, a combination of L-gulonic acid $\gamma$ lactone at $0.2 \mathrm{mg} / \mathrm{ml}$ and olive oil at $0.2 \%$ was assayed to determine whether the response enhancement caused by matrix effect can be completely corrected for all of the pesticides studied. Since good quantification results were already achieved for juice samples using olive oil as an analyte protectant, only soil and honey samples were studied, and pesticide residues were quantified employing a standard prepared with a mixture of the protectants. Figure 1B shows the good peak shape and chromatographic resolution obtained with the combination of protectants compared with the results obtained with a standard in neat solvent previously shown (Fig. 1A, dotted line). Table 4 shows the quantification results obtained for all of the pesticides in both matrices. The results are in the range of the normally accepted recoveries $(70-120 \%)$ with relative standard deviations lower than $10 \%$, despite the difficulty of simulating the matrix effect with analyte protectants. The chromatographic response was found to be linear in the studied range of concentrations (10-100 ppb). The limit of detection (LOD) for each pesticide, determined as a value three-times the background noise obtained at its retention time in a blank sample and its corresponding limit of quantification (LOQ), calculated as a value ten-times that background noise, were near $1 \mathrm{ppb}$ and lower than $10 \mathrm{ppb}$ for most of the pesticides studied, respectively.

\section{Conclusions}

In this work, 24 pesticides were analyzed by GC-MS in soil, juice and honey. Standards prepared in neat solvent were compared with standards prepared in blank matrix extracts; a noticeable matrix effect was observed. The matrix-induced chromatographic response effect depended on the matrix type, due to the type and concentration of the co-extracted compounds; it was more important in soil and honey extracts.

Four analyte protectants were evaluated for the GC-MS analysis of pesticides to counteract the enhancement of the chromatographic response. The analyte protection provided by 2,3-butanediol was lower than that of the other types of protectants studied. The obtained results showed that $0.2 \%$ of olive oil was able to counteract the matrix effect in juice samples, and that a mixture of L-gulonic acid $\gamma$-lactone $(0.2$ $\mathrm{mg} / \mathrm{ml})$ and olive oil $(0.2 \%)$ could be used for the determination of pesticide residues in soil and honey matrices. Good results were obtained with the proposed method employing analyte protectants and, therefore, the use of fortified blank samples as calibration standards can be avoided.

\section{References}

1. J. L. Tadeo, C. Sánchez-Brunete, B. Albero, and L. González, Crit. Rev. Anal. Chem., 2004, 34, 165.

2. M. Fernández, Y. Picó, and J. Mañes, J. Food Prot., 2002, 65, 1502.

3. F. J. Schenck and S. J. Lehotay, J. Chromatogr., A, 2000, $868,51$.

4. D. R. Erney, A. M. Gillespie, D. M. Gilvydis, and C. F. Poole, J. Chromatogr., 1993, 638, 57.

5. M. Godula, J. Hajslová, and K. Alterová, J. High Resol. Chromatogr., 1999, 22, 395.

6. F. J. Egea González, M. E. Hernández Torres, E. Almansa López, L. Cuadros Rodríguez, and J. L. Martínez Vidal, J. Chromatogr., A, 2002, 966, 155.

7. J. Hajslová, K. Holadová, V. Kocourek, J. Poustka, M. Codula, P. Cuhra, and M. Kempný, J. Chromatogr., A, 1998, 800, 283.

8. C. Sánchez-Brunete, B. Albero, and J. L. Tadeo, J. Agric. Food Chem., 2004, 52, 1445.

9. B. Albero, C. Sánchez-Brunete, and J. L. Tadeo, Talanta, 2005, 66, 916 . 
10. B. Albero, C. Sánchez-Brunete, and J. L. Tadeo, J. Agric. Food Chem., 2004, 52, 5828.

11. M. Anastassiades, K. Mastovska, and S. J. Lehotay, J. Chromatogr., A, 2003, 1015, 163.

12. V. Kocourek, J. Hajslová, K. Holadová, and J. Poustka, J. Chromatogr., A, 1998, 800, 297.

13. J. L. Bernal, M. J. del Nozal, J. J. Jiménez, and J. M. Rivera, J. Chromatogr., A, 1997, 778, 111.
14. D. R. Erney and C. F. Poole, J. High Resol. Chromatogr., 1993, 16, 501.

15. M. Anastassiades, S. J. Lehotay, D. Stajnbaher, and F. J. Schenck, J. AOAC Int., 2003, 86, 412.

16. J. W. Wong, M. G. Webster, C. A. Halverson, M. J. Hengel, K. K. Ngim, and S. E. Ebeler, J. Agric. Food Chem., 2003, 51, 1148. 